\title{
Maternal and Fetal Outcomes in Pregnant Patients with Active Lupus Nephritis
}

\author{
Steven J. Wagner, MD ${ }^{1}$, lasmina Craici, MD ${ }^{1}$, Darcy Reed, MD $^{2}$, Suzanne Norby, MD ${ }^{1}$, Kent \\ Bailey, PhD ${ }^{3}$, Heather J. Wiste, BA ${ }^{3}$, Christina M. Wood, MS ${ }^{3}$, Kevin G. Moder, MD ${ }^{4}$, Kimberly \\ P. Liang, MD ${ }^{4}$, Kelly V. Liang, MD ${ }^{1}$, Carl Rose, MD $^{5}$, Tomas Rozkos, MS ${ }^{6}$, Michal Sitina, \\ MD $^{6}$, Joseph P. Grande, MD, PhD $^{7}$, and Vesna D. Garovic, MD $^{1}$ \\ ${ }^{1}$ Division of Nephrology and Hypertension, Mayo Clinic \\ ${ }^{2}$ Department of Primary Care Internal Medicine, Mayo Clinic \\ ${ }^{3}$ Department of Biostatistics, Mayo Clinic, Rochester MN \\ ${ }^{4}$ Division of Rheumatology, Mayo Clinic, Rochester MN \\ ${ }^{5}$ Department of Obstetrics and Gynecology, Mayo Clinic, Rochester MN \\ ${ }^{6}$ Charles University, Hradec Kralove, Czech Republic \\ ${ }^{7}$ Department of Pathology
}

\begin{abstract}
PURPOSE-The objective of this study was to determine the impact of lupus nephritis disease activity on maternal and fetal outcomes in pregnant patients with systemic lupus erythematosus (SLE).

METHODS-Medical records of all pregnant patients with SLE treated at our institution between 1976 and 2007 were reviewed. All patients met American College of Rheumatology classification criteria for SLE. Demographic data, history of lupus nephritis, nephritis disease activity, and maternal and fetal outcomes of pregnancy were abstracted. Active lupus nephritis was defined as the presence of proteinuria $>0.5 \mathrm{~g} /$ day and/or active urinary sediment with or without an elevation in serum creatinine $(\mathrm{Cr})$. Quiescent lupus nephritis was confirmed in the presence of proteinuria $<0.5 \mathrm{mg} /$ day and inactive urinary sediment.
\end{abstract}

RESULTS-We identified fifty-eight patients with ninety pregnancies. Compared to pregnancies in SLE patients without renal involvement $(n=47)$, pregnancies in patients with active lupus nephritis $(n=23)$ were associated with a higher incidence of maternal complications $(57 \%$ vs. $11 \%, \mathrm{p}<0.001)$, whereas those with quiescent lupus nephritis $(\mathrm{n}=20)$ were not $(35 \%$ vs. $11 \%, \mathrm{p}=0.10)$. Women with active lupus nephritis were more likely to deliver preterm than women without lupus nephritis, median of 34 weeks vs. 40 gestational weeks, respectively ( $\mathrm{p}=0.002$ ), and were more likely to suffer fetal loss $(35 \%$ vs $9 \%, \mathrm{p}=0.031)$.

CONCLUSION-Active, but not quiescent, lupus nephritis during pregnancy is associated with a higher incidence of maternal and fetal complications compared to pregnancies in SLE patients without renal involvement.

Correspondence \& reprint requests: Vesna D. Garovic, MD, Division of Nephrology and Hypertension, Mayo Clinic College of Medicine, 200 First Street SW, Rochester, Minnesota 55905 USA, Tel: (507) 284-3594, Fax: (507) 284-1161, E-mail: garovic.vesna @ mayo.edu. 


\section{Keywords}

Pregnancy; Systemic lupus erythematosus; Lupus nephritis; Pregnancy outcomes; Preeclampsia

\section{Introduction}

Systemic lupus erythematosus (SLE) is an autoimmune connective tissue disorder that commonly affects women of childbearing age. Most reports agree that fertility is preserved in the absence of advanced renal insufficiency (i.e., creatinine $\geq 3 \mathrm{mg} / \mathrm{dL}$ ) and with no previous therapy with cytotoxic alkylating agents. However, substantial controversy exists with respect to the effects of SLE on pregnancy outcomes, with early reports suggesting a poor prognosis 1,2 in contrast to recent studies showing more favorable outcomes. ${ }^{3,4}$ At present, live births occur in at least $85 \%$ of SLE pregnancies. ${ }^{5}$ Published data have identified several risk factors for poor pregnancy outcomes, including hypertension and anti-phospholipid syndrome. In addition, several studies have suggested that renal involvement and the presence and degree of proteinuria at the time of conception may contribute to adverse maternal and fetal outcomes. 6,7 A multivariate analysis of 141 pregnancies in SLE patients identified lupus nephritis as the only significant predictor of fetal loss. ${ }^{8}$ However, only a few retrospective studies with relatively small numbers of patients have compared the effects on pregnancy outcomes between active (i.e., current) versus quiescent (i.e., inactive, past) lupus nephritis at the time of conception. These studies have indicated that active disease correlates with a higher risk for subsequent pregnancy complications. ${ }^{2,} 9,{ }^{10}$ In this study, we aimed to extend previously published data regarding the impact of active lupus nephritis on maternal and fetal complications by studying pregnancy outcomes in SLE patients in a single large tertiary medical center.

\section{Patients and Methods}

\section{Patients}

The medical records of all women with onset of SLE prior to pregnancy seen at our institution between 1976 and August 2007 were reviewed. The protocol was approved by the Institutional Review Board and all subjects had consented to the use of their records for research.

Pregnancies occurring before or concomitant with SLE onset were excluded. Also, pregnancies terminated electively were excluded from this analysis. Fifty-eight patients with ninety pregnancies met these criteria (Table 1). All patients fulfilled the 1997 American College of Rheumatology (ACR) classification criteria for SLE. ${ }^{11}$ Biopsies were classified according to the 1995 World Health Organization categorizations for lupus nephritis. ${ }^{12}$ Demographic data, ACR classification criteria for SLE, history of lupus nephritis, nephritis disease activity, and maternal and fetal outcomes of pregnancy were abstracted.

\section{Patient categories}

Of the 58 patients identified, 34 women with no history of lupus nephritis had 47 pregnancies. The remaining 26 women had active lupus nephritis ( 16 women, 23 pregnancies) or histories of lupus nephritis in remission at conception (11 women, 20 pregnancies), (Table 1). Three women were included in multiple study groups with their various pregnancies. Two of them had no renal involvement during their first pregnancies and then developed active lupus nephritis during their second pregnancies. The third woman had active lupus nephritis during her first pregnancy and then 2 more pregnancies after her lupus nephritis went into remission, i.e., became quiescent. 


\section{Definitions}

The following definitions were used in this study:

SLE: 1997 American College of Rheumatology criteria for classification of SLE ${ }^{11}$

Histological diagnosis of lupus nephritis: The classification was made according to the World Health Organization (WHO) categorization. ${ }^{12}$ We did not use the classification developed by the International Society of Nephrology and the Renal Pathology Society, ${ }^{13}$ as most of our patients underwent a kidney biopsy before 2004 and were classified according to the former. In addition, it allowed us to compare our results with other published studies that, for the most part, used the WHO classification.

Current (active) lupus nephritis: The presence of active urinary sediment and/or proteinuria > $0.5 \mathrm{~g} /$ day with or without elevation in serum creatinine $(\geq 1.2 \mathrm{mg} / \mathrm{dL})$ before 20 weeks gestation.

Past (inactive, quiescent) lupus nephritis: Proteinuria $<0.5 \mathrm{mg} / \mathrm{day}$, inactive urinary sediment

Active urinary sediment: $>5$ red and white blood cells per high power field and/or $\geq 1$ cellular cast

$\underline{\text { Renal insufficiency: Serum Cr level } \geq 1.2 \mathrm{mg} / \mathrm{dL}}$

Lupus nephritis flare: Urinary sediment turning active from inactive with an increase in serum Cr by $\geq 30 \%$, or worsening proteinuria, defined as an increase by $2 \mathrm{~g} / 24$ hours if baseline proteinuria was $<3.5 \mathrm{~g} / 24$ hours, or doubling of proteinuria in patients who previously had a nephrotic range proteinuria 9

Preterm birth or delivery: Live birth before 37 weeks gestation

Spontaneous abortion: Spontaneous loss of a fetus before 20 weeks gestation

Stillbirth: Intrauterine fetal demise at $\geq 20$ weeks of gestation

Therapeutic abortion: Termination of pregnancy for medical indications

Elective abortion: Voluntary termination of pregnancy

Neonatal death: Death of a neonate before 28 days following birth

Intrauterine growth retardation (IUGR): Estimated birth weight less than $10^{\text {th }}$ percentile for gestational age

Hypertension: Chronic hypertension was based on a well-documented diagnosis of blood pressure $>140 / 90 \mathrm{~mm} \mathrm{Hg}$ before 20 weeks gestation

Gestational hypertension: Defined as either a systolic blood pressure $\geq 140 \mathrm{~mm} \mathrm{Hg}$ or a diastolic blood pressure $\geq 90 \mathrm{~mm} \mathrm{Hg}$ on at least two occasions, at least 6 hours apart after 20 weeks gestation

Preeclampsia: Onset of hypertension after 20 weeks gestation as manifested by elevated blood pressures $\geq 140 / 90 \mathrm{~mm} \mathrm{Hg}$ on two occasions, at least 6 hours apart, in previously normotensive patients, accompanied by new-onset proteinuria $\geq 300 \mathrm{mg} / \mathrm{day}$, in patients without proteinuria at baseline. Other criteria supporting a diagnosis of preeclampsia included an elevated serum 
alanine aminotransferase (ALT) concentration (> 70 units/L), increasing proteinuria in patients with pre-existing renal disease, persistent severe headaches, or epigastric pain.

Eclampsia: Generalized convulsions and/or coma in the setting of preeclampsia, and in the absence of other neurological conditions

HELLP: Syndrome of hemolysis, elevated liver enzymes, and low platelet count (thrombocytopenia) during pregnancy

\section{Laboratory Methods}

Serum chemistries, liver function tests, lupus serologies, urine protein, and blood cell counts, including platelet counts, were measured according to standardized laboratory procedures. Renal biopsies were obtained percutaneously by core needle biopsy. Specimens were analyzed under light microscopy using hematoxylin and eosin (H \& E) stain, periodic acid Schiff's (PAS) stain, methenamine silver stain, and trichrome stain; immunofluorescent microscopy using antibodies directed against immunoglobulin (Ig) G, IgA, IgM, complement C3, C1q, albumin, fibrinogen, kappa, and lambda light chains; and electron microscopy. Lupus nephritis was classified histologically by experienced renal pathologists based on WHO categorization criteria. $^{12}$

\section{Statistical analysis}

Descriptive data were summarized overall or within group by mean $\pm \mathrm{SD}$, median (25th percentile, 75th percentile) or by frequency (percent). Due to low numbers of pregnancies in both the lupus nephritis sub-categories (active $n=23$, and inactive $n=20$ ), clinical outcomes were compared between patients with either active or inactive lupus nephritis and those with SLE, but without renal involvement (control group). Twenty-six subjects had two pregnancies or more; in order to account for within-mother correlation of outcomes, the group differences were tested for equality to zero by a bootstrap approach, in which bootstrap samples of mothers were selected, and all of their pregnancies included each time they appeared in the bootstrap sample, and the group differences recalculated. The proportion of such samples in which the group differences were inconsistent in sign with the observed result was multiplied by 2 to obtain a 2 -sided p-value. Differences were considered significant when this p-value was $<0.05$.

\section{Results}

\section{Lupus nephritis}

Among 90 pregnancies affected by SLE, there were 43 pregnancies with and 47 pregnancies without lupus nephritis. Of the 43 with lupus nephritis, 23 had active disease, while 20 had no evidence of activity at their first prenatal visit, which typically occurred during the first trimester of their pregnancies. A greater number of pregnancies with any history of lupus nephritis were treated with prednisone, antimalarials, or azathioprine during pregnancy $(n=39$, $91 \%)$ compared to those without a history of lupus nephritis $(n=19,40 \%), p<0.001$. There was no difference in medication use between active and quiescent lupus nephritis. Women with active lupus nephritis were younger at delivery than women with SLE but without renal involvement. No patient received cyclophosphamide during pregnancy. The prevalence of renal insufficiency was not statistically significant between the groups. Lupus nephritis was histologically confirmed in 19 of 26 patients. Histological subtypes according to WHO classifications included 1 mesangial, 8 focal proliferative, 7 diffuse proliferative and 3 membranous. 


\section{Fetal Outcomes and lupus nephritis}

Active lupus nephritis resulted in greater fetal loss (35\% vs $9 \% \mathrm{p}=0.031$ ) compared to pregnancies in women without lupus nephritis, and in more frequent preterm delivery (52\% vs $19 \%, \mathrm{p}=0.007$ ), median gestational age at delivery 34 and 40 weeks, respectively (Table 2). No differences in fetal loss and gestational age at delivery were noted between women with quiescent lupus nephritis and those without lupus nephritis (36.5 weeks vs 40 weeks). There was not a statistically significant difference in intrauterine growth restriction between any of the groups. No cases of neonatal death or neonatal lupus were seen.

\section{Maternal outcomes and lupus nephritis}

There was no difference in method of delivery between groups. Women with active lupus nephritis experienced a higher rate of obstetrical complications, including hypertension during pregnancy, preeclampsia, eclampsia, stroke, HELLP syndrome, and maternal death, compared with controls, i.e., women with SLE, but without any history of renal involvement $(p<0.001)$ (Table 3). The rates of maternal complications were not significantly different in women with inactive lupus nephritis compared to SLE without renal involvement $(\mathrm{p}=0.10)$. Finally, two of 20 patients $(10 \%)$ with quiescent lupus at conception developed a renal flare in pregnancy. The first patient developed an elevation in $\mathrm{Cr}$ from 1.0 to $1.6 \mathrm{mg} / \mathrm{dL}$ in the first trimester with worsening of proteinuria. A kidney biopsy was performed that showed the features of both diffuse proliferative (class IV) and membranous (class V) lupus nephritis. She had positive anti-phospholipid antibodies and histories of deep venous thrombosis and two first-trimester miscarriages. She was treated with low-molecular weight heparin, prednisone $60 \mathrm{mg}$ daily, and hydroxychloroquine $200 \mathrm{mg}$ daily. She died 3 weeks after the kidney biopsy from respiratory failure, presumably due to pulmonary hemorrhage/embolism. The second patient achieved lupus nephritis remission on azathioprine and remained in remission throughout her pregnancy. She delivered a stillborn infant at 36 weeks of gestation. Her Cr increased from 0.8 to $1.2 \mathrm{mg} /$ dL within first month postpartum; she was diagnosed with and treated for a lupus nephritis flare.

\section{Discussion}

Our study demonstrates an association between active lupus nephritis at conception and complications of pregnancy, including preterm delivery, fetal loss, gestational hypertension, preeclampsia, eclampsia, stroke, HELLP syndrome, and maternal death. The difference in pregnancy outcomes appears not to be related to the prevalence of renal insufficiency, as this was not different between the groups. Similar to previous studies, ${ }^{14}$ we have observed no significant differences in pregnancy outcomes between women with quiescent lupus nephritis at conception and those with SLE, but without renal involvement. Our results extend previous reports by confirming the association between adverse pregnancy outcomes and active lupus nephritis in a single center cohort, in which we were able to confirm pregnancy complications on one side, and to apply strict diagnostic criteria for SLE and lupus nephritis activity on the other. This was possible due to our record keeping system of all patients' laboratory results, histopathology findings, and consultation notes, which enabled us to obtain detailed clinical information about our patients despite the retrospective character of our study. Our data further support the current recommendation that, in order to optimize pregnancy outcomes in SLE patients, conception should occur during an interval of disease quiescence. A recent review of this topic ${ }^{15}$ suggested that patients should be in remission for at least $12-18$, and not 6 months as proposed by most authors. Our study further indicates that pregnancy outcomes are similar between SLE patients with either no renal involvement or quiescent lupus nephritis. This is in agreement with a study from Italy that identified quiescence of renal disease at the onset of pregnancy to be the only predictor of favorable maternal outcomes in women with lupus nephritis. ${ }^{9}$ 
The rate of prematurity seen in this study (48\%) extends previous observations that prematurity is a common complication in SLE pregnancies, and further increased with renal involvement (30-58\%). ${ }^{9,16,17}$ The frequencies of gestational hypertension, preeclampsia/eclampsia, and fetal loss found in our study are similar to previous studies. ${ }^{6,9}$ We used a predefined set of diagnostic criteria in order to distinguish lupus nephritis flare from preeclampsia superimposed on preexisting renal disease and proteinuria. However, the differential diagnosis between these two conditions in the absence of a definitive method is often difficult. ${ }^{18}$ The presence of extrarenal SLE manifestations, abnormal serologies coupled with decreasing complement levels, active urinary sediment, and response to prednisone would make the diagnosis of a lupus nephritis flare more likely. In the future, more sensitive methods to assess glomerular damage may be advantageous in this setting. For example, the presence of podocyturia has preliminarily been shown to be a sensitive and specific marker of preeclampsia. ${ }^{19}$ But, the presence of podocyturia has been demonstrated in clinically active lupus nephritis in one human study, with the treatment of the lupus nephritis with corticosteroids resulting in disappearance of podocyturia. ${ }^{20}$ In animal studies, podocyturia appears to be a marker of ongoing, active glomerular injury. ${ }^{21}$ Ultimately, development of new technologies for the specific and timely diagnosis of preeclampsia, as well as of flares of lupus nephritis, in pregnant patients with SLE may lead to innovative therapeutic modalities for these conditions in hopes of improving maternal and fetal outcomes in women with SLE.

Our study has several limitations. It spanned a period of 31 years during which new medications were developed (such as mycophenolate mofetil) that might have influenced the control of SLE activity and thus pregnancy outcomes in these patients. Due to a relatively small sample, we were unable to differentiate among different lupus nephritis classes, which previously have been shown to influence pregnancy outcomes. ${ }^{22}$ Specifically, hypertension and preeclampsia were more prevalent in patients with lupus nephritis classes III and IV, compared to those with lupus nephritis classes II and V. Finally, 27 patients in our series had 2 or more pregnancies. A bias might have been introduced by the fact that women who had favorable pregnancy outcomes may more readily decide to conceive again than those with adverse pregnancy outcomes. In addition, a previous study indicated that, in the same mother, sequential, multiple pregnancy outcomes are highly correlated. ${ }^{23}$ However, we were able to account for withinmother correlation in outcomes through the implementation of the bootstrap statistical method.

It is important to note that we defined lupus nephritis disease activity early in pregnancy, i.e., at the first pregnancy visit, which typically occurred during the first trimester, thus likely representing disease activity at the time of conception. Ten percent of our patients with quiescent lupus at conception developed a subsequent renal flare, either later in pregnancy or during the postpartum period. Results from previous reports are controversial, with some studies reporting renal flares in as many as $60 \%$ of SLE patients, ${ }^{5}$ and others arguing that pregnancy does not cause exacerbation of renal disease in SLE. ${ }^{24}$ The heterogeneity of the study designs, with different controls being used by different studies, has been identified as one of the possible reasons for the conflicting nature of published results. ${ }^{5}$ Our sample size was too small to adequately address this question. Future studies of renal disease activity are needed that will be adequately powered to study these associations in a prospective manner.

The findings of our study underscore the importance of pre-pregnancy counseling of women with lupus nephritis. According to our data and previously published studies, SLE patients with active lupus nephritis are at higher risk for pregnancy complications than SLE patients without renal disease, and should be advised against pregnancy until a renal remission of at least 6 months duration, if not 12-18 according to most recent recommendations, has been achieved. Women with quiescent disease should be counseled that their pregnancy risks are likely not different from those of SLE patients without renal involvement, assuming that their renal function has not been significantly affected by the previous disease process. 


\section{References}

1. Bear R. Pregnancy and lupus nephritis. A detailed report of six cases with a review of the literature. Obstet Gynecol 1976;47(6):715-718. [PubMed: 934563]

2. Le Thi Huong D, Wechsler B, Piette JC, Bletry O, Godeau P. Pregnancy and its outcome in systemic lupus erythematosus. QJM 1994;87(12):721-729. [PubMed: 7859048]

3. Wong CH, Chen TL, Lee CS, Lin CJ, Chen CP. Outcome of pregnancy in patients with systemic lupus erythematosus. Taiwan J Obstet Gynecol 2006;45(2):120-123. [PubMed: 17197351]

4. Derksen RH, Bruinse HW, de Groot PG, Kater L. Pregnancy in systemic lupus erythematosus: a prospective study. Lupus 1994;3(3):149-155. [PubMed: 7951299]

5. Petri M. Hopkins Lupus Pregnancy Center: 1987 to 1996. Rheum Dis Clin North Am 1997;23(1):113. [PubMed: 9031371]

6. Huong DL, Wechsler B, Vauthier-Brouzes D, Beaufils H, Lefebvre G, Piette JC. Pregnancy in past or present lupus nephritis: a study of 32 pregnancies from a single centre. Ann Rheum Dis 2001;60(6): 599-604. [PubMed: 11350849]

7. Johns KR, Morand EF, Littlejohn GO. Pregnancy outcome in systemic lupus erythematosus (SLE): a review of 54 cases. Aust N Z J Med 1998;28(1):18-22. [PubMed: 9544381]

8. Rahman P, Gladman DD, Urowitz MB. Clinical predictors of fetal outcome in systemic lupus erythematosus. J Rheumatol 1998;25(8):1526-1530. [PubMed: 9712095]

9. Moroni G, Quaglini S, Banfi G, et al. Pregnancy in lupus nephritis. Am J Kidney Dis 2002;40(4):713720. [PubMed: 12324905]

10. Rahman FZ, Rahman J, Al-Suleiman SA, Rahman MS. Pregnancy outcome in lupus nephropathy. Arch Gynecol Obstet 2005;271(3):222-226. [PubMed: 15052490]

11. Hochberg MC. Updating the American College of Rheumatology revised criteria for the classification of systemic lupus erythematosus. Arthritis Rheum 1997;40(9):1725. [PubMed: 9324032]

12. Churg, J. New York: Igaku Shoin; 1995. Renal diseases, classification and atlas of glomerular diseases.

13. Weening JJ, D'Agati VD, Schwartz MM, et al. The classification of glomerulonephritis in systemic lupus erythematosus revisited. J Am Soc Nephrol 2004;15(2):241-250. [PubMed: 14747370] [comment][erratum appears in J Am Soc Nephrol. 2004 Mar;15(3):835-6].

14. Julkunen H, Jouhikainen T, Kaaja R, et al. Fetal outcome in lupus pregnancy: a retrospective casecontrol study of 242 pregnancies in 112 patients. Lupus 1993;2(2):125-131. [PubMed: 8330034]

15. Kong NC. Pregnancy of a lupus patient--a challenge to the nephrologist. Nephrology Dialysis Transplantation 2006;21(2):268-272.

16. Imbasciati E, Surian M, Bottino S, et al. Lupus nephropathy and pregnancy. A study of 26 pregnancies in patients with systemic lupus erythematosus and nephritis. Nephron 1984;36(1):46-51. [PubMed: 6691001]

17. Cavallasca JA, Laborde HA, Ruda-Vega H, Nasswetter GG. Maternal and fetal outcomes of 72 pregnancies in Argentine patients with systemic lupus erythematosus (SLE). Clin Rheumatol 2008;27 (1):41-46. [PubMed: 17516127]

18. Petri M. The Hopkins Lupus Pregnancy Center: Ten Key Issues in Management. Rheumatic Disease Clinics of North America 2007;33(2):227-235. [PubMed: 17499704]

19. Garovic VD, Wagner SJ, Turner ST, et al. Urinary podocyte excretion as a marker for preeclampsia. [see comment]. Am J Obstet Gynecol 2007;196(4):e1-e7. [PubMed: 17403404]320

20. Nakamura T, Ushiyama C, Suzuki S, et al. Urinary podocytes for the assessment of disease activity in lupus nephritis. Am J Med Sci 2000;320(2):112-116. [PubMed: 10981486]

21. Yu D, Petermann A, Kunter U, Rong S, Shankland SJ, Floege J. Urinary podocyte loss is a more specific marker of ongoing glomerular damage than proteinuria. J Am Soc Nephrol 2005;16(6):17331741. [PubMed: 15829708]

22. Carmona F, Font J, Moga I, et al. Class III-IV proliferative lupus nephritis and pregnancy: a study of 42 cases. Am J Reprod Immunol 2005;53(4):182-188. [PubMed: 15760379] 
23. Ramsey-Goldman R, Kutzer JE, Kuller LH, Guzick D, Carpenter AB, Medsger TA Jr. Previous pregnancy outcome is an important determinant of subsequent pregnancy outcome in women with systemic lupus erythematosus. Am J Reprod Immunol 1992;28(3-4):195-198. [PubMed: 1285877]

24. Lockshin MD, Reinitz E, Druzin ML, Murrman M, Estes D. Lupus pregnancy. Casecontrol prospective study demonstrating absence of lupus exacerbation during or after pregnancy. Am J Med 1984;77(5):893-898. [PubMed: 6496544] 


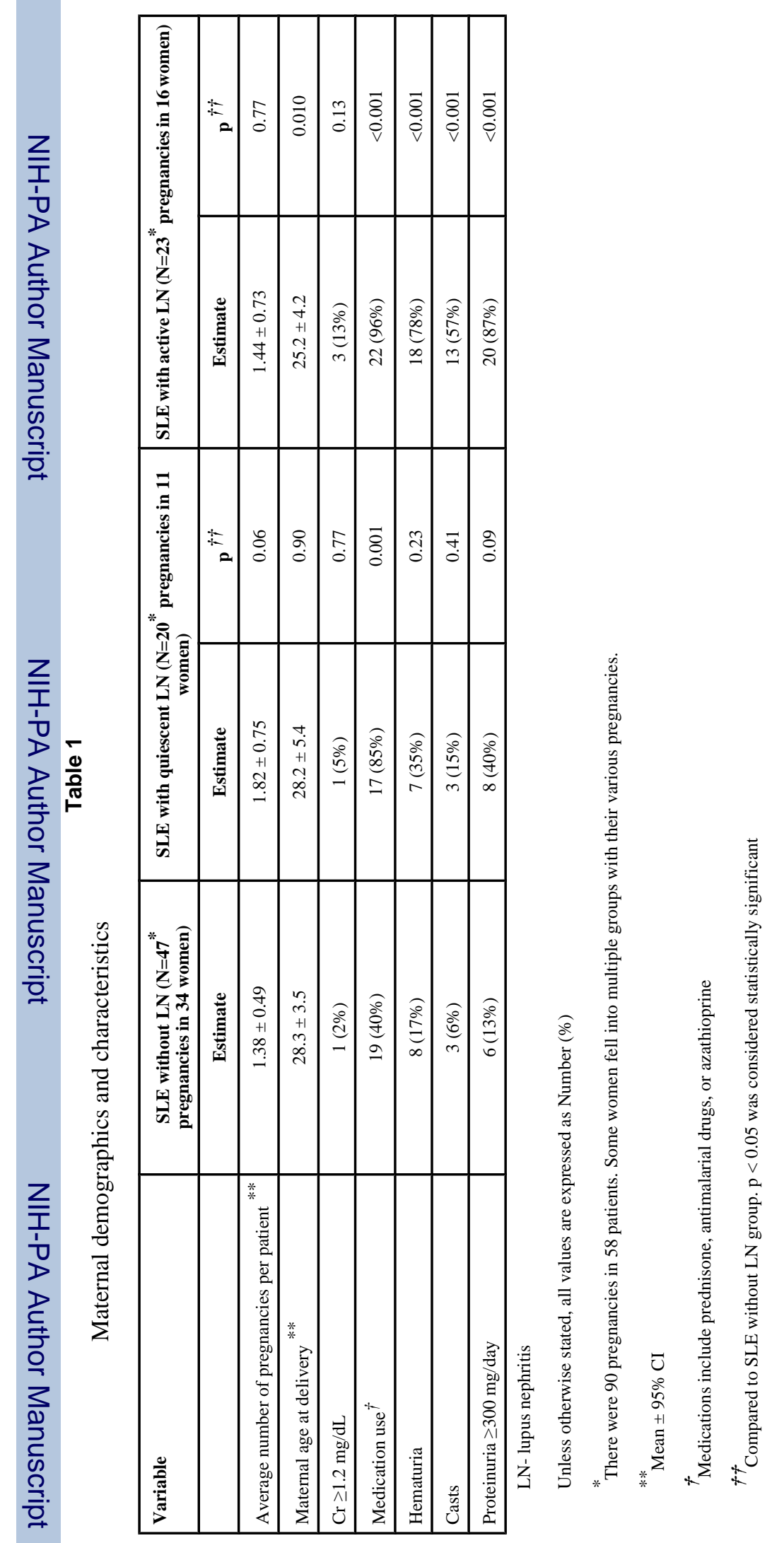




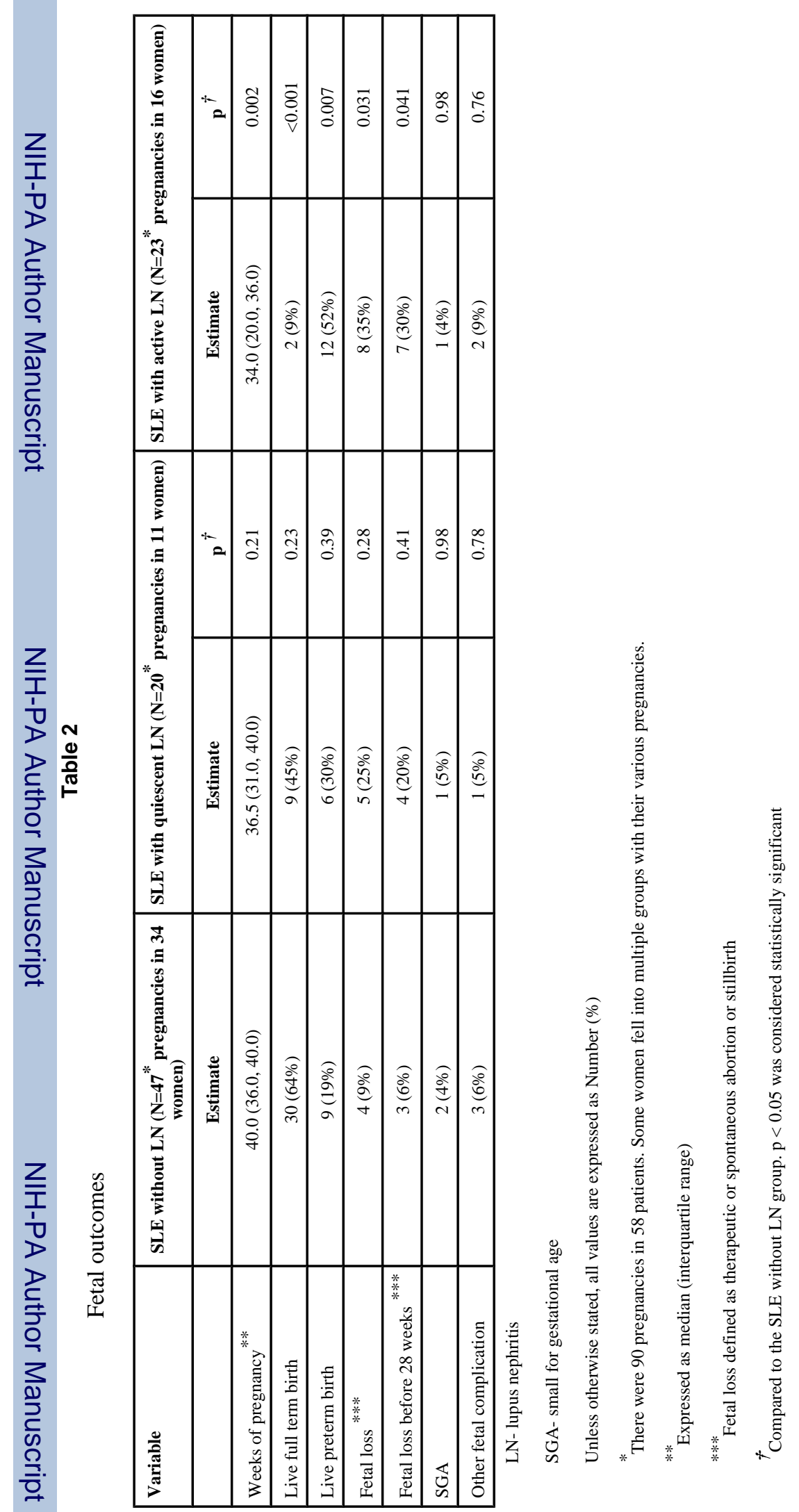

Lupus. Author manuscript; available in PMC 2009 August 11. 


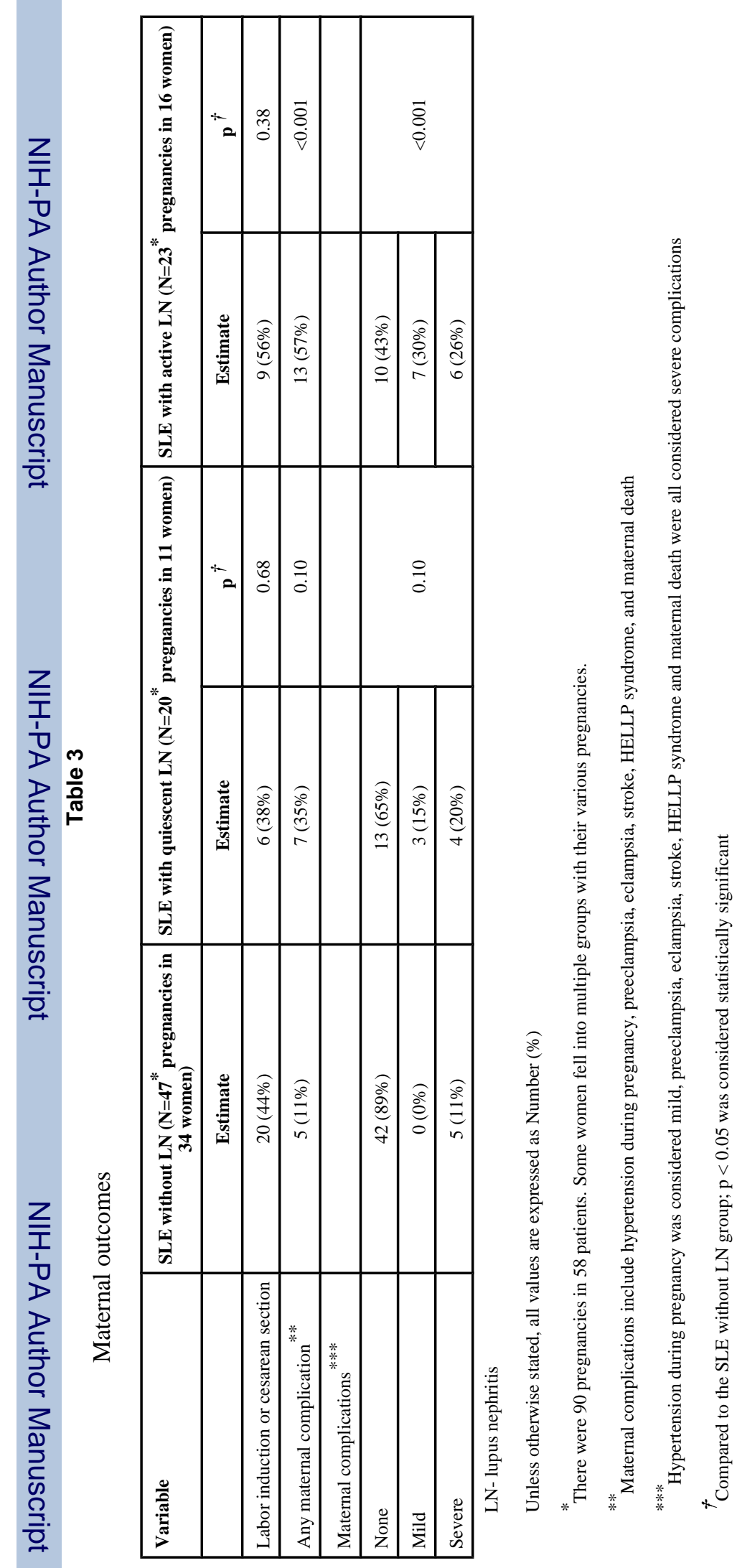

Lupus. Author manuscript; available in PMC 2009 August 11. 